\title{
Twistronics: A Recent Avenue in van der Waals Heterostructures ${ }^{\dagger}$
}

\author{
Pramoda Kumar Nayak ${ }^{1, *}$ \\ 1 Department of Physics and Materials Science Research Centre, Indian Institute of Technology Madras, Chennai 600036, \\ India \\ * Correspondence: pnayak@iitm.ac.in; \\ $\dagger$ Presented at Materials Chemistry and Physics (Materials Chemistry 2020) - International e-Conference
}

Received: 16.09.2020; Revised: 20.09.2020; Accepted: 24.09.2020; Published: 27.09.2020

\begin{abstract}
Two-dimensional (2D) materials, the thinnest form of materials to ever occur in nature, have the potential to dramatically alter and revolutionize our material world. After the discovery of graphene, the most prominent representative of this class of materials, many other $2 \mathrm{D}$ crystals have been identified. Even if individual 2D materials own various interesting and unexpected properties, the stacking of such layers leads to 'artificial vdW solids' called van der Waals heterostructures (vdW HSs) that result in the emergence of new states of matter with novel functionalities. The vdW HSs not only depend on the combination of different 2D crystal but also on their rotational alignment opening the avenue for a new field called twistronics. Coupling between the two layers depends on the stacking angle, which can be used as an external degree of freedom to tune their optical and electronic properties. Apart from excitonic ground states, 2D transition metal dichalcogenides (TMDs) and their heterostructures offer an excellent platform to explore fascinating higher-order excitations such as trion, biexciton, interlayer exciton, hybrid exciton, moiré exciton, and so on. The emergence of these higherorder excitations mostly depends on the symmetry, temperature, and the band alignment of the heterobilayer systems.
\end{abstract}

Keywords: twistronics; 2D materials; vdW heterostructures; higher-order excitations; inter-layer excitons; hybrid exciton; Moiré exciton.

(C) 2020 by the authors. This article is an open-access article distributed under the terms and conditions of the Creative Commons Attribution (CC BY) license (https://creativecommons.org/licenses/by/4.0/).

\section{Funding}

This research was funded by the Department of Science and Technology, Government of India (DST-GoI), with sanction order no. SB/S2/RJN-043/2017 under Ramanujan Fellowship.

\section{Acknowledgments}

This research has no acknowledgment.

\section{Conflicts of Interest}

The authors declare no conflict of interest. 